\title{
Comammox-a newly discovered nitrification process in the terrestrial nitrogen cycle
}

\author{
Hang-Wei Hu ${ }^{1,2} \cdot$ Ji-Zheng $\mathrm{He}^{1}$ (i)
}

Received: 11 September 2017 / Accepted: 10 October 2017 / Published online: 18 October 2017

(C) Springer-Verlag GmbH Germany 2017

\begin{abstract}
Purpose Nitrification, the microbial oxidation of ammonia to nitrate via nitrite, is a pivotal component of the biogeochemical nitrogen cycle. Nitrification was conventionally assumed as a two-step process in which ammonia oxidation was thought to be catalyzed by ammonia-oxidizing archaea (AOA) and bacteria (AOB), as well as nitrite oxidation by nitrite-oxidizing bacteria (NOB). This long-held assumption of labour division between the two functional groups, however, was challenged by the recent unexpected discovery of complete ammonia oxidizers within the Nitrospira genus that are capable of converting ammonia to nitrate in a single organism (comammox). This breakthrough raised fundamental questions on the niche specialization and differentiation of comammox organisms with other canonical nitrifying prokaryotes in terrestrial ecosystems.

Materials and methods This article provides an overview of the recent insights into the genomic analysis, physiological characterization and environmental investigation of the comammox organisms, which have dramatically changed our perspective on the aerobic nitrification process. By using quantitative PCR analysis, we also compared the abundances of comammox Nitrospira clade A and clade B, AOA, AOB and NOB in 300 forest soil samples from China spanning a wide range of soil $\mathrm{pH}$.
\end{abstract}

Responsible editor: Zhihong Xu

Ji-Zheng He

jzhe@rcees.ac.cn

1 Faculty of Veterinary and Agricultural Sciences, The University of Melbourne, Parkville, VIC 3010, Australia

2 State Key Laboratory of Urban and Regional Ecology, Research Center for Eco-Environmental Sciences, Chinese Academy of Sciences, Beijing 100085, China
Results and discussion Comammox Nitrospira are environmentally widespread and numerically abundant in natural and engineered habitats. Physiological data, including ammonia oxidation kinetics and metabolic versatility, and comparative genomic analysis revealed that comammox organisms might functionally outcompete other canonical nitrifiers under highly oligotrophic conditions. These findings highlight the necessity in future studies to re-evaluate the niche differentiation between ammonia oxidizers and their relative contribution to nitrification in various terrestrial ecosystems by including comammox Nitrospira in such comparisons.

Conclusions The discovery of comammox and their broad environmental distribution added a new dimension to our knowledge of the biochemistry and physiology of nitrification and has far-reaching implications for refined strategies to manipulate nitrification in terrestrial ecosystems and to maximize agricultural productivity and sustainability.

Keywords Ammonia oxidation - Comammox · Complete nitrification $\cdot$ Niche separation $\cdot$ Nitrite oxidation $\cdot$ Nitrospira

\section{The discovery of comammox: a paradigm shift}

The microbial oxidation of ammonia $\left(\mathrm{NH}_{3}\right)$ via nitrite $\left(\mathrm{NO}_{2}{ }^{-}\right)$to nitrate $\left(\mathrm{NO}_{3}{ }^{-}\right)$, termed nitrification, is a central process of the biogeochemical nitrogen (N) cycle (Fig. 1) (Prosser 2011). The two consecutive nitrification steps, ammonia oxidation $\left(\mathrm{NH}_{3} \rightarrow\right.$ $\left.\mathrm{NO}_{2}{ }^{-}\right)$and nitrite oxidation $\left(\mathrm{NO}_{2}^{-} \rightarrow \mathrm{NO}_{3}{ }^{-}\right)$, were traditionally thought to be catalyzed by two physiologically distinct clades of ammonia-oxidizing bacteria (AOB) and nitrite-oxidizing bacteria (NOB), respectively, whose close cooperation is essential for the complete conversion of ammonia to nitrate (Costa et al. 2006). Since the discovery of ammonia-oxidizing archaea (AOA) as a second major type of ammonia oxidizers (Könneke et al. 2005), 
Fig. 1 Major processes of the nitrogen cycle. The classical nitrification was assumed as a two-step process performed by two functional groups of organisms: ammonia oxidizers (AOA and $\mathrm{AOB}$ ) and nitriteoxidizing bacteria (NOB). Recent studies have shown that comammox Nitrospira can conduct both steps of nitrification, and novel substrates can be used for the generation of ammonium. (Abbreviations: DNRA, dissimilatory reduction to ammonium)

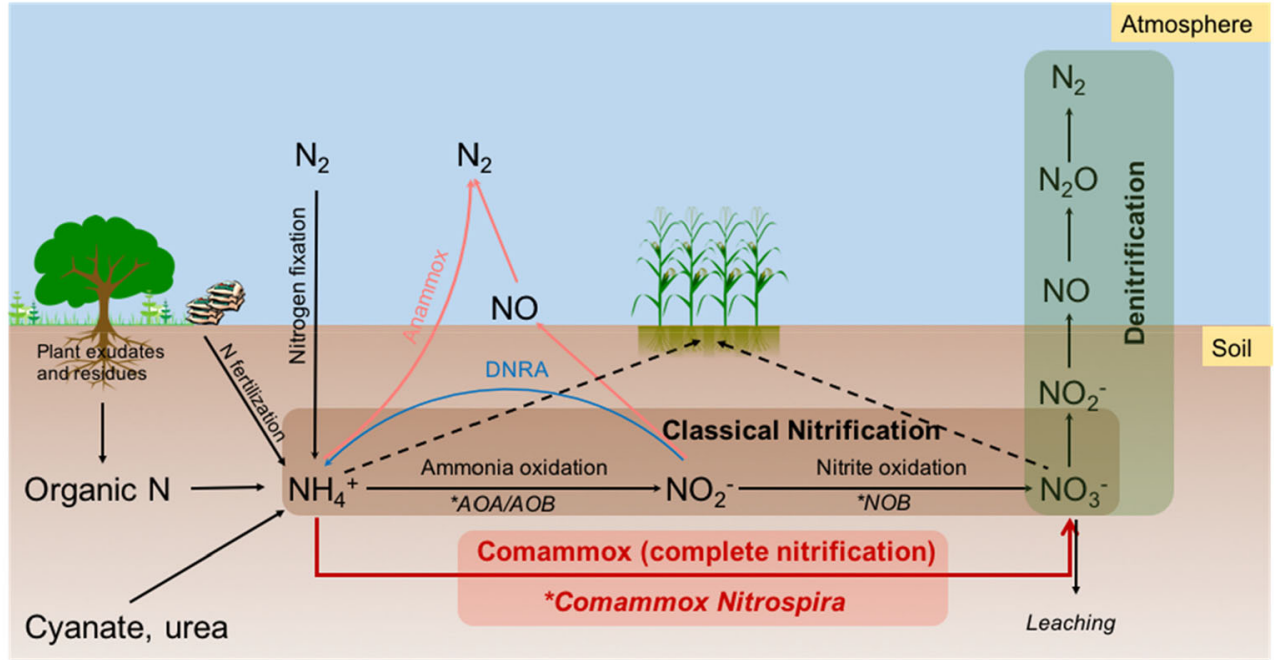

nitrification research has received an impetus towards unravelling the niche specialization and ecological significance of AOA that are ubiquitous and abundant in a wide variety of terrestrial ecosystems (Leininger et al. 2006; He et al. 2007, 2012; Prosser and Nicol 2012; Hu et al. 2014). Although the relative contribution of $\mathrm{AOA}$ and $\mathrm{AOB}$ to nitrification is still in debate, increasing evidence supports their niche separation on the basis of the physiological, cellular and genomic differences between AOA and AOB - the high efficiency of metabolism and high substrate affinity suits an oligotrophic lifestyle of AOA in acidic soils and Nlimited environments (He et al. 2007, 2012; Lehtovirta-Morley et al. 2011; Zhang et al. 2012; Hu et al. 2015b). In addition to their functional importance in nitrification, AOA can also substantially contribute to formation of nitrous oxide $\left(\mathrm{N}_{2} \mathrm{O}\right)$ (Stieglmeier et al. 2014; Hink et al. 2016) - a potent greenhouse gas and a major ozone-depleting substance (Hu et al. 2015a, 2017). By contrast, NOB, including members of Nitrospira, Nitrobacter and Nitrococcus genera, have received less attention though they have been repeatedly reported that abundance of Nitrospira exceeded those of AOA and AOB in various ecosystems (Foesel et al. 2008; Gruber-Dorninger et al. 2015).

The catalysis of the two nitrification steps by two distinct groups of organisms - ammonia oxidizers and NOB-has been a central hypothesis in nitrification research for over a century (Costa et al. 2006). The close coupling of ammonia oxidation and nitrite oxidation was used to explain the frequently observed co-occurrence of ammonia oxidizers with NOB in nitrifying consortia (Arp and Bottomley 2006). This functional separation, however, has puzzled scientists for a long time because complete oxidation of ammonia to nitrate in one organism was theoretically assumed to be energetically advantageous compared with separated nitrification steps (Costa et al. 2006). Therefore, a single organism with lower growth rates but higher growth yields than canonical ammonia oxidizers was postulated to be present in nature to perform both nitrification steps (Costa et al. 2006). These hypothetical complete nitrifiers (termed as "comammox"_complete ammonia oxidizers) might have a competitive advantage over canonical ammonia oxidizers and NOB in ammoniadepleted biofilms, microbial aggregates, soils and sediments, where comammox do not have to share the energy derived from nitrification with a partner organism (Costa et al. 2006; Kuypers 2015; Nunes-Alves 2016).

This hypothesis was recently proved to be true by the successful enrichment of the bacterial organisms capable of complete nitrification from a deep oil well (Daims et al. 2015) and an aquaculture system (van Kessel et al. 2015) through growing biofilm samples supplied continuously with low concentrations of ammonium. In contrast to known ammonia oxidizers and NOB, the assembled complete genomes of comammox Nitrospira harbour the full set of genes encoding ammonia monooxygenase (AMO) and hydroxylamine dehydrogenase (HAO) for ammonia oxidation, and genes encoding nitrite oxidoreductase (NXR) for nitrite oxidation (Daims et al. 2015; van Kessel et al. 2015; Camejo et al. 2017), indicative of their genetic potential for complete nitrification. The comammox organisms enriched from both studies were restricted to the widely distributed lineage II of the genus Nitrospira, suggesting that comammox Nitrospira might be common nitrifiers in terrestrial and aquatic ecosystems (Daims et al. 2015, 2016). The presence of comammox might help to explain some previous observations, for example (i) the usually low nitrite concentrations in the environment if comammox Nitrospira do not release nitrite as an intermediate during complete nitrification and (ii) the high abundances of Nitrospira bacteria, often exceeding AOA and AOB, if some of these Nitrospira are actually belonging to comammox (Wang et al. 2015; Daims et al. 2016).

These findings have fundamentally changed our perspective on the microbial regulation of the aerobic nitrification process and point to comammox Nitrospira as a novel and important nitrifying component with a potentially 
underappreciated role in the biogeochemical $\mathrm{N}$ cycle (Kuypers 2015; Nunes-Alves 2016; Santoro 2016). Considering the potential ecological importance of comammox and our limited knowledge on this novel group of ammonia oxidizers, the biochemical and physiological characterizations of the enriched comammox organisms, the environmental investigation of comammox and the exploration of their relative contribution to nitrification in various ecosystems will be a new focus of nitrification research in a near future.

\section{The environmental relevance of comammox Nitrospira}

The discovery of the novel metabolic capacity of complete nitrification in the Nitrospira genus has prompted questions regarding their ecological significance and environmental relevance in diverse ecosystems. Metagenomic screening of environmental samples in many published databases, based on the functional gene sequences for ammonia oxidation (amoA, amoB, amoC and hao genes) in the enriched comammox species, revealed the widespread occurrence of putative comammox Nitrospira in a range of terrestrial and aquatic ecosystems including agricultural soils, freshwater habitats, wastewater treatment plants (WWTPs) and drinking water treatment systems (Daims et al. 2015; van Kessel et al. 2015; Pinto et al. 2015; Palomo et al. 2016; Bartelme et al. 2017; Wang et al. 2017) (Table 1). The comammox amoA gene sequences are highly similar to some previously assigned methane monooxygenase sequences of uncultured methanotrophs belonging to Crenothrix polyspora (Daims et al. 2015; van Kessel et al. 2015). Therefore, previous PCR-based studies might have mistakenly classified the comammox amoA gene into the Crenothrix pmoA gene in environmental samples (Stoecker et al. 2006). These findings provide strong indications that comammox Nitrospira might have much broader environmental distribution than previously thought and have to be incorporated into future studies of nitrification in these ecosystems.

To facilitate further investigation of the abundance, diversity and distribution of comammox, cultivation-independent approaches such as quantitative PCR are needed to more efficiently detect and quantify comammox Nitrospira in environmental samples. The subunit A of AMO (AmoA) of comammox Nitrospira forms a distinct cluster that is clearly separated from AOA and AOB amoA genes, suggesting that the $a m o A$ gene can be a suitable phylogenetic marker for targeting comammox organisms (Pjevac et al. 2017). Comammox Nitrospira were placed within two monophyletic sister clades, clade A and clade B, based on phylogeny of comammox amoA genes (Daims et al. 2015; van Kessel et al. 2015). The new PCR primer sets that can specifically target the comammox amoA clade A and clade B was developed and validated in a recent study (Pjevac et al. 2017). By using the newly designed primer, a considerable diversity of comammox organisms harbouring unique amoA gene was detected in engineered systems, rice paddy soils, forest soils, rice rhizosphere and brackish lake sediment (Pjevac et al. 2017). Comammox amoA clade A comprised $14 \sim 34 \%$ of the total amoA gene abundance in the activated sludge from a WWTP, indicating that comammox could be an important contributor to nitrification in this engineered system (Pjevac et al. 2017). The comparable abundances of comammox amoA genes and those of AOA and AOB amoA genes in forest and rice paddy soils indicate that comammox organisms are also functionally relevant in terrestrial ecosystems (Pjevac et al. 2017). We further used this primer set to quantify the abundance of comammox, AOA, AOB and NOB in 300 forest soil samples collected from five forest biomes in China (Wang et al. 2016). It was found that both clades of comammox Nitrospira were highly abundant in these forest soils and more dominant than AOB and NOB across the entire gradient of soil pH (Fig. 2). The abundances of comammox clade A and AOA were comparable in alkaline soils, but comammox clade A was more abundant than AOA in acidic soils (Fig. 2). Similarly, comammox Nitrospira were the most abundant ammonia oxidizers (approximately 1.9-fold of AOA) in a biofilter (Bartelme et al. 2017), while AOA were 3.8- to 10.5-fold more abundant than comammox Nitrospira in three metagenomic datasets from the Rifle soils (Wrighton et al. 2012; Daims et al. 2015). The widespread occurrence of comammox Nitrospira is consistent with the previous findings that some uncharacterized Nitrospira groups were present in various habitats (Ke et al. 2013; Pester et al. 2014). Although we are just beginning to understand the environmental relevance of comammox, these findings have important implications for the global $\mathrm{N}$ cycle, particularly if these organisms are functionally active in the complete nitrification. With the increasing number of comammox sequences retrieved from the environment, further refined primers with higher coverage will be required to better capture comammox Nitrospira from the terrestrial ecosystem.

\section{Niche separation between comammox Nitrospira and canonical nitrifiers}

The discovery of comammox Nitrospira as a new competitor for the limited ammonia sources in soils and their cooccurrence with AOA and AOB call into re-evaluation of the niche partitioning and the relative contribution of comammox and other nitrifying prokaryotes to nitrification (Santoro 2016). Although the potential contribution of comammox to nitrification in terrestrial ecosystems remains completely untapped, physiological data, including ammonia 
Table 1 Studies reporting the occurrence of comammox Nitrospira in various ecosystems

\begin{tabular}{|c|c|c|c|}
\hline Environments & Techniques & Major findings & References \\
\hline $\begin{array}{l}\text { A recirculation aquaculture } \\
\text { system }\end{array}$ & $\begin{array}{l}\text { Enrichment of microbial } \\
\text { consortium, genome } \\
\text { sequencing, fluorescence } \\
\text { in situ hybridization }\end{array}$ & $\begin{array}{l}\text { Two enriched Nitrospira species ( } N \text {. nitrosa } \\
\text { and } N \text {. nitrificans) encode all the enzymes } \\
\text { necessary for ammonia oxidation via nitrite } \\
\text { to nitrate in their genomes and indeed } \\
\text { completely oxide ammonium to nitrate in } \\
\text { incubation experiments. }\end{array}$ & van Kessel et al. (2015) \\
\hline $\begin{array}{l}\text { Soil, sediment, WWTP } \\
\text { sludge, air, water, } \\
\text { microbial mat, coral reef }\end{array}$ & $\begin{array}{l}\text { Metagenome/metatranscriptome } \\
\text { screening of Nitrospira-like } \\
\text { amoA sequences on MG-RAST }\end{array}$ & $\begin{array}{l}\text { A total of } 168 \text { metagenomes and } 28 \\
\text { metatranscriptomes contained at least two } \\
\text { reads affiliated with comammox amoA group, } \\
\text { yielding a total of } 3727 \text { reads that were } \\
\text { obtained mainly from soil, sediments and } \\
\text { wastewater treatment plants }\end{array}$ & van Kessel et al. (2015) \\
\hline A $1200-m$ deep oil well & $\begin{array}{l}\text { Enrichment of microbial } \\
\text { consortium, genome } \\
\text { sequencing, fluorescence } \\
\text { in situ hybridization }\end{array}$ & $\begin{array}{l}\text { A completely nitrifying bacterium (N. inopinata) } \\
\text { from the genus Nitrospira was discovered and } \\
\text { possessed the full genetic complement for both } \\
\text { ammonia and nitrite oxidation. }\end{array}$ & Daims et al. (2015) \\
\hline $\begin{array}{l}\text { Soils, paddy field } \\
\text { floodwater, freshwater } \\
\text { and engineered } \\
\text { environments }\end{array}$ & $\begin{array}{l}\text { Metagenomic screening of } \\
\text { public databases }\end{array}$ & $\begin{array}{l}\text { Sequences within comammox AmoA clade A } \\
\text { were retrieved from natural and engineered } \\
\text { environments, but no comammox marker } \\
\text { genes were identified in marine environments. }\end{array}$ & Daims et al. (2015) \\
\hline $\begin{array}{l}\text { Drinking water treatment } \\
\text { plant }\end{array}$ & Shotgun DNA sequencing & $\begin{array}{l}\text { This study provided metagenomics evidence } \\
\text { for the presence of a Nitrospira-like organism } \\
\text { with the metabolic potential for comammox in } \\
\text { a drinking water system. }\end{array}$ & Pinto et al. (2015) \\
\hline $\begin{array}{l}\text { Wastewater treatment } \\
\text { bioreactors }\end{array}$ & Tag-454-pyrosequencing & $\begin{array}{l}\text { Phylogenetic analysis revealed that a few of } \\
\text { the Nitrospira sequences found in the } \\
\text { bioreactors were comammox. }\end{array}$ & $\begin{array}{l}\text { Gonzalez-Martinez et al. } \\
\quad \text { (2016) }\end{array}$ \\
\hline $\begin{array}{l}\text { An oligotrophic engineered } \\
\text { system }\end{array}$ & $\begin{array}{l}\text { Shotgun metagenomic } \\
\text { sequencing }\end{array}$ & $\begin{array}{l}\text { The amo-containing Nitrospira genome contigs } \\
\text { were identified, suggesting the potential for } \\
\text { complete ammonium oxidation by Nitrospira. }\end{array}$ & Palomo et al. (2016) \\
\hline $\begin{array}{l}\text { A full-scale hybrid biofilm } \\
\text { and activated sludge } \\
\text { reactor }\end{array}$ & $\begin{array}{r}\text { Metagenomic analysis and } \\
\text { tag-454-pyrosequencing }\end{array}$ & $\begin{array}{l}\text { Nitrospira-like amoA genes were identified, } \\
\text { suggesting the presence of comammox with } \\
\text { potential to perform complete nitrification. }\end{array}$ & Chao et al. (2016) \\
\hline $\begin{array}{l}\text { Atmospheric fine particulate } \\
\text { matter }\end{array}$ & $\begin{array}{l}\text { Quantitative PCR, cloning } \\
\text { and sequencing }\end{array}$ & $\begin{array}{l}\text { Comammox co-exist with AOA and AOB in } \\
\text { the atmosphere, with a potential important } \\
\text { role in ammonia oxidation in } \mathrm{PM}_{2.5} \text {. }\end{array}$ & Gao et al. (2016) \\
\hline Drinking water systems & $\begin{array}{l}\text { Metagenomic analysis and } \\
\text { metagenomics mining }\end{array}$ & $\begin{array}{l}\text { The comammox amoA-like sequencing was } \\
\text { detected in several drinking water systems, } \\
\text { and outnumbered the AOA and AOB } \\
\text { amoA-like sequences. }\end{array}$ & Wang et al. (2017) \\
\hline $\begin{array}{l}\text { Recirculating aquaculture } \\
\text { systems }\end{array}$ & $\begin{array}{l}\text { Quantitative PCR, clone library } \\
\text { and phylogenetic analysis }\end{array}$ & $\begin{array}{l}\text { Comammox and nitrite-oxidizing Nitrospira } \\
\text { populations co-exist in the aquaculture system } \\
\text { with relatively equivalent and stable abundances. }\end{array}$ & Bartelme et al. (2017) \\
\hline $\begin{array}{l}\text { Natural and engineered } \\
\text { habitats }\end{array}$ & Quantitative PCR & $\begin{array}{l}\text { A considerable diversity of comammox amoA gene } \\
\text { was detected in engineered systems, rice paddy } \\
\text { soils, forest soils, rice rhizosphere and brackish } \\
\text { lake sediment. }\end{array}$ & Pjevac et al. (2017) \\
\hline
\end{tabular}

oxidation kinetics of comammox and metabolic versatility, and comparative genomic analysis of the enriched comammox species may improve our understanding of the niche separation between comammox and canonical nitrifiers.

\subsection{Differences in ammonia oxidation kinetics}

Previous studies have regarded differences in ammonia oxidation kinetics as a major driver of niche differentiation between AOA and AOB (Martens-Habbena et al. 2009; Prosser and Nicol 2012; Hu et al. 2014). Physiological analysis of the first AOA strain, Nitrosopumilus maritimus SCM1, revealed an apparent half-saturation constant $\left(K_{\mathrm{m}(\mathrm{app})}\right)$ for $\mathrm{NH}_{3}$ of approximately $3 \mathrm{nM}$, which is by now the lowest reported $K_{\mathrm{m}(\mathrm{app})}$ among all the cultured ammonia oxidizers (MartensHabbena et al. 2009) (Fig. 3). The pure comammox bacterium, Nitrosopumilus inopinata, had a high ammonia affinity as revealed by a $K_{\mathrm{m}(\mathrm{app})}$ for $\mathrm{NH}_{3}$ of approximately $63 \mathrm{nM}$, which is 4- to 2500-fold below values reported for AOB (Kits et al. 2017). The mean $K_{\mathrm{m}(\mathrm{app})}$ values for $\mathrm{NH}_{3}(410$ to $703 \mathrm{nM})$ of 
Fig. 2 Abundances of amoA genes from comammox Nitrospira, $\mathrm{AOA}$ and $\mathrm{AOB}$ and $n x r B$ gene of NOB in 300 forest soil samples with soil $\mathrm{pH}$ ranging from 4.0 to 8.6. Quantitative PCR analysis of comammox amoA clade A and clade B was performed using the primer sets and thermal cycling conditions as described previously (Pjevac et al. 2017). The basic information of the 300 forest soils was given in Wang et al. (2016)

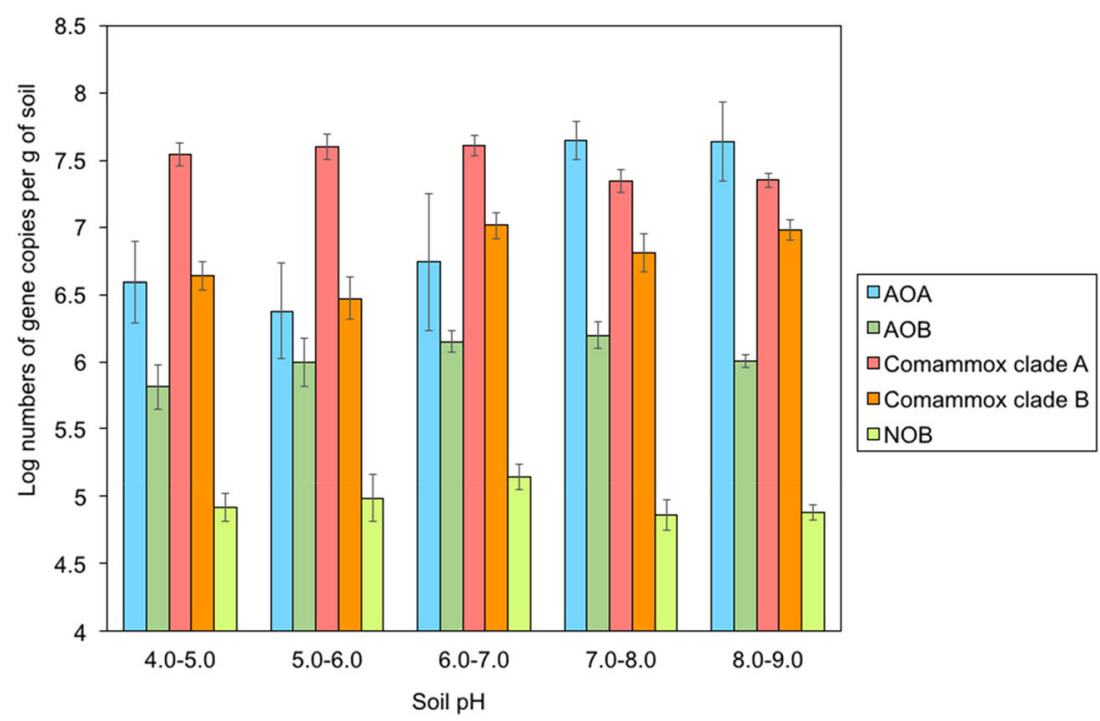

the two AOA isolates belonging to the Nitrososphaera cluster: $N$. viennensis from soil (Tourna et al. 2011) and $N$. gargensis from a hot spring (Palatinszky et al. 2015) are remarkably higher than those of $N$. inopinata and $N$. maritimus SCM1 (Kits et al. 2017). The AOA enrichments belonging to the Nitrosopumilus cluster-Nitrosotenuis uzonensis from a hot spring (Lebedeva et al. 2013) and the enrichment " $5 \mathrm{~A}$ " of a Ca. $N$. uzonensis (Kits et al. 2017) — have mean $K_{\mathrm{m} \text { (app) values }}$ for $\mathrm{NH}_{3}$ at $5200 \mathrm{nM}$ and $870 \sim 960 \mathrm{nM}$, respectively, which are significantly higher than those of $N$. inopinata and N. maritimus SCM1 (Kits et al. 2017). However, the reported
$K_{\mathrm{m} \text { (app) }}$ values for $\mathrm{NH}_{3}(\sim 20$ to $40 \mathrm{nM})$ of two AOA enrichment cultures of Ca. Nitrosoarchaeum koreensis from marine sediments and agricultural soils (Park et al. 2010; Jung et al. 2011) are comparable to that of comammox N. inopinata (Fig. 3). The affinity of AOA for $\mathrm{NH}_{3}$ is not consistently higher than that of AOB. For example, the $K_{\mathrm{m}(\mathrm{app})}$ values for $\mathrm{NH}_{3}$ (1770 to $4120 \mathrm{nM}$ ) of oligotrophic AOB (including Nitrosomonas cluster 6a G5-7, Nitrosomonas oligotropha, and Nitrosospira briensis ATCC 25971) are within the range

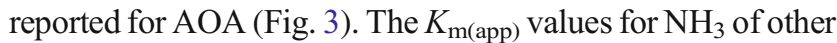
characterized AOB cultures ranged from 7380 to $61,000 \mathrm{nM}$,
Fig. 3 Comparison of the apparent half-saturation constant $\left(K_{\mathrm{m}(\mathrm{app})}\right)$ for ammonia $\left(\mathrm{NH}_{3}\right)$ (in red colour) across the comammox strain $N$. inopinata, $\mathrm{AOA}$ and AOB strains, as well as the calculated ammonia concentrations in published soil microcosm studies (in blue colour), where $\mathrm{AOA}$ or $\mathrm{AOB}$ were found to be functionally predominant (Hu et al. 2014)

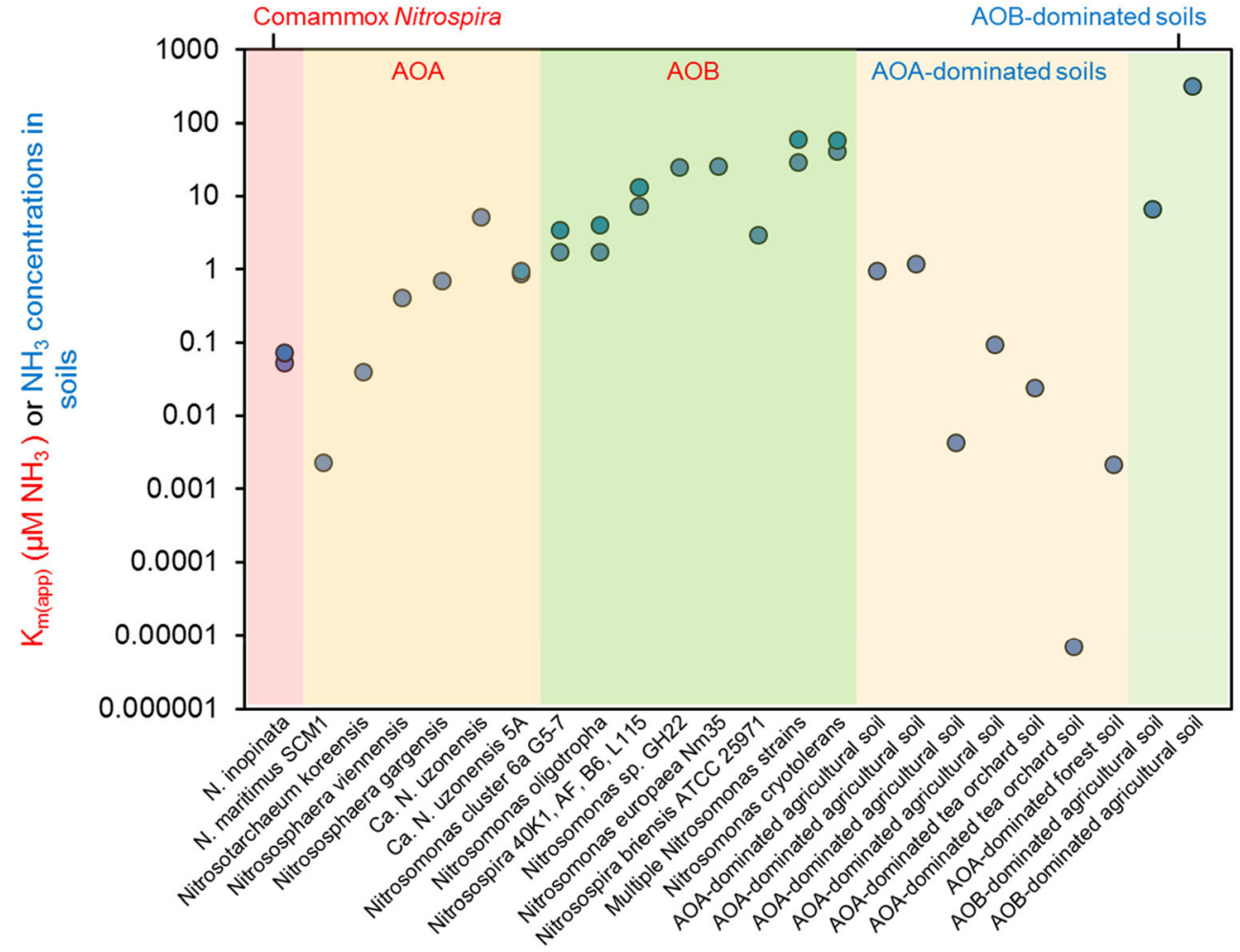


which are significantly higher than those of comammox N. inopinata and AOA (Fig. 3).

This comparison of the ammonia oxidation kinetics for comammox $N$. inopinata, AOA and AOB cultures from various habitats suggested that, in contrast to earlier perceptions, AOA are not necessarily the most competitive ammonia oxidizers for ammonia substrate under oligotrophic conditions. The overlap between the $K_{\mathrm{m}}$ (app) values of comammox $N$. inopinata and kinetically characterized AOA isolates (Fig. 3) suggests that comammox may co-exist and directly compete with oligotrophic AOA in niches characterized by low ammonia availability, such as drinking water treatment systems, groundwater wells and freshwater biofilters (Daims et al. 2015; Pinto et al. 2015; Palomo et al. 2016; Bartelme et al. 2017; Wang et al. 2017) and forest soils where comammox amoA gene abundances outnumbered the AOA and $\mathrm{AOB}$ amoA sequences (Fig. 2). By contrast, comammox Nitrospira were in very low abundances in active sludge and biofilm samples where substrate is not limited (Chao et al. 2016), indicating a potential minor role of comammox in these habitats. As shown in Fig. 3, the $K_{\mathrm{m} \text { (app) }}$ values of comammox $N$. inopinata were within the range of calculated ammonia concentrations in soil microcosms functionally dominated by AOA but were orders of magnitude lower than those in soil microcosms dominated by AOB. These findings demonstrate that the relative contribution of ammonia oxidizers to nitrification should be re-evaluated by including comammox as a third group of nitrifier particularly in soils where nitrification was previously thought to be carried out by AOA. However, it should be noted that $N$. inopinata remains the only kinetically characterized comammox species by now. Cultivationdependent studies are required to obtain more representative comammox strains from different environments and to determine their ammonia oxidation kinetics.

\subsection{Metabolic versatility}

Apart from the different affinity for ammonia, the genomic potential for alternative energy sources may also contribute to the niche differentiation of comammox and canonical ammonia oxidizers. Genomic analysis revealed that the enriched comammox Nitrospira species (Daims et al. 2015; van Kessel et al. 2015) encoded urease proteins and the corresponding $\mathrm{ABC}$ transport systems, enabling the use of urea as an alternative ammonia source for energy and biosynthesis. The comammox genomes retrieved from a rapid sand filter harbour a diversity of urea transporters, including high affinity urea $\mathrm{ABC}$ transporters (urtABCDE), an urea carboxylaserelated transporter $(u c t T)$ and an outer-membrane porin $(f m d C)$ involved in uptake of short-chain amides and urea at extremely low concentrations (Palomo et al. 2017). Another comammox-like genome also included the $u r e A B C D F G$ urease operon and the $\operatorname{urtABCDE}$ urea transport system, indicating a high-affinity uptake system for urea (Camejo et al. 2017). This trait may enable comammox organisms to thrive in habitats with low or fluctuating urea concentrations, such as fertilized agricultural soils, WWTPs and many aquatic and terrestrial systems. However, the genes encoding urease and urea transporter have been also found in isolated AOA strains $N$. viennensis (Tourna et al. 2011) and $N$. gargensis (Spang et al. 2012), and some strains of AOB (Burton and Prosser 2001), as well as in metagenomics investigation of polar Thaumarchaeota (Alonso-Sáez et al. 2012). AOA harbouring the putative $u r e C$ genes (encoding the alpha subunit of an archaeal urease) were reported to be metabolically active and predominate the aerobic nitrification in ureaamended agricultural soils (Lu and Jia 2013). These findings suggest that comammox organisms would directly compete with $\mathrm{AOA}$ and $\mathrm{AOB}$ for the urea substrate in the environment particularly under ammonia-poor conditions.

Other traits or metabolic versatility, apart from the ureolytic pathway, may also influence the niche partitioning and specialization of nitrifiers depending on the environmental conditions. These included the following: (1) the whole genome sequence analysis of $N$. inopinata suggests extensive metabolic versatility including hydrogen and sulphide oxidation and fermentative metabolism (Kits et al. 2017); (2) the genes coding for formate dehydrogenase are present in comammox clade B but not in comammox clade A, providing an opportunity for niche differentiation in oxic-anoxic transition zones where formate is frequently observed as a product of fermentation (Koch et al. 2015; Palomo et al. 2017); (3) comparative genomic analysis revealed that cyanate hydratase genes are only detected in NOB Nitrospira, which may benefit NOB Nitrospira over comammox Nitrospira in cyanate-containing environments through cyanate degradation and nitrification by reciprocal feeding with ammonia oxidizers (Palatinszky et al. 2015; Camejo et al. 2017); (4) the genomes of comammox Nitrospira but not AOA or AOB contain an alkaline phosphatase, which is highly expressed under phosphorus limiting conditions, suggesting that comammox have potential to compete under phosphorous limitation and starvation (Palomo et al. 2017); (5) comammox Nitrospira harbour the enzymes and pathways involved in degradation of various carbon compounds suggesting that they have the potential to grow mixotrophically (Palomo et al. 2017); and (6) a lowaffinity Rh-type ammonium transporter is only present in clade A comammox genomes, while a high-affinity AmtBtype transporter was detected in clade B and canonical Nitrospira genomes (Palomo et al. 2017).

\subsection{Soil pH}

Soil $\mathrm{pH}$ has been recognized as a major factor controlling the biogeographic distribution and metabolic activity of ammonia oxidizers in terrestrial environments (Nicol et al. 2008; He 
et al. 2012; Prosser and Nicol 2012; Hu et al. 2013). Ammonia $\left(\mathrm{NH}_{3}\right)$ rather than ammonium ion $\left(\mathrm{NH}_{4}{ }^{+}\right)$has been generally considered as the direct substrate for ammonia oxidizers, and acidic soil is characterized by low ammonia availability due to the ionization of ammonia by hydrogen ions (Hu et al. 2014). We used quantitative PCR analysis of functional genes to compare the abundances of $\mathrm{AOA}, \mathrm{AOB}, \mathrm{NOB}$ and comammox clade A and clade B in 300 forest soil samples across a wide range soil $\mathrm{pH}$ from 4.0-8.6 (Fig. 2). Clade A comammox were the most abundant nitrifier in acidic soils $(\mathrm{pH}<6.0)$, followed by clade B comammox and AOA, while the abundances of AOB and NOB were relatively low in acidic forest soils (Fig. 2). These findings suggest that comammox organisms might also be an important contributor to nitrification in acidic soils, challenging our previous notion that AOA, rather than $\mathrm{AOB}$, make a major contribution to nitrification in acidic soils (He et al. 2012; Hu et al. 2014). However, it should be noted that DNA/RNA-stable isotope probing technique, nitrification inhibitor and transcriptional analysis are needed to provide evidence of the nitrification activity of comammox organisms in acidic soils. High-throughput sequencing is further required to demonstrate the distribution patterns of different comammox lineages along the gradient of soil $\mathrm{pH}$ and to identify the distinct $\mathrm{pH}$-adapted populations within comammox. Differences in adaptation to soil $\mathrm{pH}$ might also be an important factor leading to niche partitioning between the comammox organisms and other nitrifiers.

\section{Research priorities for comammox Nitrospira in terrestrial ecosystems}

Given the environmental prevalence and potential importance of comammox, we outlined a core set of research priorities to facilitate our understanding of the role of comammox organisms in the terrestrial $\mathrm{N}$ cycle.

\section{Enrichment, isolation and physiological characterization} of comammox species from the soil environment All the available comammox cultures were obtained from aquatic ecosystems, and the lack of comammox cultures from the terrestrial environment has hampered our understanding of the ecological role of comammox in soils. With the development of advanced cultivation techniques, single-cell genome sequencing and metagenomics, comammox isolates from the terrestrial environment can provide opportunities to experimentally characterize their species-specific ammonia oxidation kinetics and nitrification rates, which will serve as a basis for a better understanding of the microbial mechanisms underpinning the niche separation between comammox and canonical nitrifiers.
Quantification of the relative contribution of comammox and canonical nitrifiers to nitrification in various terrestrial ecosystems It is critical to understand the extent to which comammox contribute to nitrification in a wide range of terrestrial ecosystems by conducting controlled microcosm and pot experiments. The combined use of DNA/RNA-stable isotope probing (Zhang et al. 2012; Hu et al. 2015b), quantitative PCR, omics-based approaches and nitrification inhibitors may allow distinguishing the contribution of different nitrifying groups to nitrification. Previous incubation experiments have shown that $N$. inopinata (Daims et al. 2015) and the other two comammox species ( $N$. nitrosa and $N$. nitrificans) (van Kessel et al. 2015) are capable of inorganic carbon fixation during complete nitrification. Therefore, ${ }^{13} \mathrm{CO}_{2}$-DNA/RNA-stable isotope probing technique can be employed to measure the metabolic activity of comammox and quantify their contribution to nitrification.

Potential microbial production of $\mathrm{N}_{2} \mathrm{O}$ by comammox organisms in terrestrial ecosystems It remains an outstanding question whether comammox organisms can produce $\mathrm{N}_{2} \mathrm{O}$ (Santoro 2016). Given the similarity of their ammonia oxidation pathway to that of classic AOB, it is possible that comammox may be also involved in $\mathrm{N}_{2} \mathrm{O}$ production. Incubation studies can provide correlative evidence regarding the relationships between the abundance and the diversity of comammox with $\mathrm{N}_{2} \mathrm{O}$ fluxes, but direct evidence will come from the physiological characterization of the pure cultures of comammox in near future.

Effects of synthetic nitrification inhibitors and/or biological nitrification inhibitors on the growth and activity of comammox organisms Incubation experiments have shown that ammonia-dependent carbon fixation of comammox Nitrospira was strongly inhibited by the addition of allylthiourea (a potent inhibitor of ammonia oxidation) (van Kessel et al. 2015). It is imperative to investigate the responses of comammox to nitrification inhibitors, such as 3,4dimethylpyrazole phosphate (DMPP) (Shi et al. 2016a, b, 2017), from a practical perspective to promote $\mathrm{N}$ use efficiency and reduce $\mathrm{N}$ losses through greenhouse gas $\mathrm{N}_{2} \mathrm{O}$ emissions and nitrate leaching from soils in agriculture.

Large-scale investigation of comammox organisms in terrestrial ecosystems and the influencing environmental factors A systematic survey of comammox and other canonical nitrifiers in various terrestrial ecosystems is urgently needed to assess the prevalence and distribution patterns of the major comammox lineages shaped by abiotic and biotic factors at regional, national and global scales. The integration of comammox in these studies, combined with omics-based approaches (metagenomics, transcriptomics, metabolomics, and proteomics) and environmental data, will be crucial to obtain a 
panoramic view of the niche specialization and niche partitioning of comammox, $\mathrm{AOB}, \mathrm{AOA}$ and NOB in terrestrial ecosystems.

\section{Concluding remarks and future perspectives}

The discovery of comammox and their broad environmental distribution has profoundly expanded our mechanistic understanding of the biochemistry and regulation of complete nitrification and has far-reaching implications for future studies on the microbial ecology of the terrestrial $\mathrm{N}$ cycle. All available information points to the highly oligotrophic habitats as the fundamental niches for comammox Nitrospira, due to their high substrate affinity for ammonia, comparatively low ammonium oxidation rate and high growth yield compared to canonical nitrifiers. More insights into the niche specialization, population dynamics, metabolic versatility, diversity and phylogenetic structure, interactions of comammox and other nitrifying prokaryotes are required before a general conclusion can be obtained on the ecological significance of comammox in terrestrial ecosystems. It will be essential to understand how comammox organisms respond to increasing loads of $\mathrm{N}$ fertilizers and deposition, anthropogenic perturbations and global climate changes such as climate warming, elevated $\mathrm{CO}_{2}$ and extreme weather events. We envision that an improved knowledge in this field may lead to refined strategies to manipulate the $\mathrm{N}$ cycling processes, in particular nitrification, and to maximize crop $\mathrm{N}$ use efficiency and agricultural productivity in agriculture.

Funding information This work was financially supported by Natural Science Foundation of China (41230857) and the Australian Research Council (DE150100870; DP160101028).

\section{References}

Alonso-Sáez L, Waller AS, Mende DR, Bakker K, Farnelid H, Yager PL et al (2012) Role for urea in nitrification by polar marine Archaea. Proc Natl Acad Sci U S A 109:17989-17994

Arp D, Bottomley PJ (2006) Nitrifier: more than 100 years from isolation to genome sequences. Microbe 1:229-234

Bartelme RP, McLellan SL, Newton RJ (2017) Freshwater recirculating aquaculture system operations drive biofilter bacterial community shifts around a stable nitrifying consortium of ammonia-oxidizing archaea and comammox Nitrospira. Front Microbiol 8:101

Burton SAQ, Prosser JI (2001) Autotrophic ammonia oxidation at low pH through urea hydrolysis. Appl Environ Microbiol 67:2952-2957

Camejo PY, Domingo JS, McMahon KD, Moguera DR (2017) Genomeenabled insights into the ecophysiology of the comammox bacterium Candidatus Nitrospira nitrosa. mSystems. https://doi.org/10. 1128/mSystems.00059-17

Chao Y, Mao Y, Yu K, Zhang T (2016) Novel nitrifiers and comammox in a full-scale hybrid biofilm and activated sludge reactor revealed by metagenomics approach. Appl Microbiol Biotechnol 100:82258237
Costa E, Perez J, Kreft JU (2006) Why is metabolic labour divided in nitrification? Trends Microbiol 14:213-219

Daims H, Lebedeva EV, Pjevac P, Han P, Herbold C, Albertsen M et al (2015) Complete nitrification by Nitrospira bacteria. Nature 528: 504-509

Daims H, Lucker S, Wagner M (2016) A new perspective on microbes formerly known as nitrite-oxidizing bacteria. Trends Microbiol 24: $699-712$

Foesel BU, Gieseke A, Schwerner C, Stief P, Koch L, Cytryn E et al (2008) Nitrosomonas Nm143-like ammonia oxidizers and Nitrospira marina-like nitrite oxidizers dominate the nitrifier community in a marine aquaculture biofilm. FEMS Microbiol Ecol 63: 192-204

Gao JF, Fan XY, Pan KL, Li HY, Sun LX (2016) Diversity, abundance and activity of ammonia-oxidizing microorganisms in fine particulate matter. Sci Rep 6:38785

Gonzalez-Martinez A, Rodriguez-Sanchez A, van Loosdrecht MCM, Gonzalez-Lopez J, Vahala R (2016) Detection of comammox bacteria in full-scale wastewater treatment bioreactors using tag-454pyrosequencing. Environ Sci Pollut Res 23:25501-25511

Gruber-Dorninger C, Pester M, Kitzinger K, Savio DF, Loy A, Rattei T, Wagner M, Damis H (2015) Functionally relevant diversity of closely related Nitrospira in activated sludge. ISME J 9:643-655

He JZ, Shen JP, Zhang LM, Zhu YG, Zheng YM, Xu MG, Di H (2007) Quantitative analyses of the abundance and composition of ammonia-oxidizing bacteria and ammonia-oxidizing archaea of a Chinese upland red soil under long-term fertilization practices. Environ Microbiol 9:2364-2374

He JZ, Hu HW, Zhang LM (2012) Current insights into the autotrophic thaumarchaeal ammonia oxidation in acidic soils. Soil Biol Biochem 55:146-154

Hink L, Nicol GW, Prosser JI (2016) Archaea produce lower yields of $\mathrm{N}_{2} \mathrm{O}$ than bacteria during aerobic ammonia oxidation in soil. Environ Microbiol. https://doi.org/10.1111/1462-2920.13282

Hu HW, Zhang LM, Dai Y, Di HJ, He JZ (2013) pH-dependent distribution of soil ammonia oxidizers across a large geographical scale as revealed by high-throughput pyrosequencing. J Soils Sediments 13: 1439-1449

Hu HW, Xu ZH, He JZ (2014) Ammonia-oxidizing archaea play a predominant role in acid soil nitrification. Adv Agron 125:261-302

Hu HW, Chen D, He JZ (2015a) Microbial regulation of terrestrial nitrous oxide formation: understanding the biological pathways for prediction of emission rates. FEMS Microbiol Rev 39:729-749

Hu HW, Macdonald CA, Trivedi P, Holmes B, Bodrossy L, He JZ, Singh BK (2015b) Water addition regulates the metabolic activity of ammonia oxidizers responding to environmental perturbations in dry subhumid ecosystems. Environ Microbiol 17:444-461

Hu HW, Trivedi P, He JZ, Singh BK (2017) Microbial nitrous oxide emissions in dryland ecosystems: mechanisms, microbiome and mitigation. Environ Microbiol. https://doi.org/10.1111/1462-2920. 13795

Jung MY, Park SJ, Min D, Kim JS, Rijpstra WIC, Dameste JSS, Kim GJ, Madsen EL, Rhee SK (2011) Enrichment and characterization of an autotrophic ammonia-oxidizing archaeon of mesophilic crenarchaeal group I.1a from an agricultural soil. Appl Environ Microbiol 77:8635-8647

Ke X, Angel R, Lu Y, Conrad R (2013) Niche differentiation of ammonia oxidizers and nitrite oxidizers in rice paddy soil. Environ Microbiol 15:2275-2292

Kits KD, Sedlacek CJ, Lebedeva EV, Han P, Bulaev A, Pjevac P et al (2017) Kinetic analysis of a complete nitrifier reveals an oligotrophic lifestyle. Nature. https://doi.org/10.1038/nature23679

Koch H, Lucker S, Albertsen M, Kitzinger K, Herbold C, Spieck E et al (2015) Expanded metabolic versatility of ubiquitous nitriteoxidizing bacteria from the genus Nitrospira. Proc Nat Acad Sci USA 112:11371-11376 
Könneke M, Bernhard AE, Jose R, Walker CB, Waterbury JB, Stahl DA (2005) Isolation of an autotrophic ammonia-oxidizing marine archaeon. Nature 437:543-546

Kuypers MMM (2015) Microbiology: a division of labour combined. Nature 528:487-488

Lebedeva EV, Hatzenpichler R, Pelletier E, Schuster N, Hauzmayer S, Bulaev A et al (2013) Enrichment and genome sequence of the group I.1a ammonia-oxidizing archaeon " $\mathrm{Ca}$. Nitrosotenuis uzonensis" representing a clade globally distributed in thermal habitats. PLoS One 8:e80835

Lehtovirta-Morley LE, Stoecker K, Vilcinskas A, Prosser JI, Nicol GW (2011) Cultivation of an obligate acidophilic ammonia oxidizer from a nitrifying acid soil. Proc Natl Acad Sci U S A 108:15892-15897

Leininger S, Urich T, Schloter M, Schwark L, Qi J, Nicol G, Prosser JI, Schuster SC, Schleper C (2006) Archaea predominate among ammonia-oxidizing prokaryotes in soils. Nature 442:806-809

Lu L, Jia Z (2013) Urea gene-containing archaea dominate autotrophic ammonia oxidation in two acid soils. Environ Microbiol 15:17951809

Martens-Habbena W, Berube PM, Urakawa H, de la Torre JR, Stahl DA (2009) Ammonia oxidation kinetics determine niche separation of nitrifying Archaea and bacteria. Nature 461:976-979

Nicol GW, Leininger S, Schleper C, Prosser JI (2008) The influence of soil $\mathrm{pH}$ on the diversity, abundance and transcriptional activity of ammonia oxidizing archaea and bacteria. Environ Microbiol 10: 2966-2978

Nunes-Alves C (2016) Do it yourself nitrification. Nat Rev Microbiol 14:61

Palatinszky M, Herbold C, Jehmlich N, Pogoda M, Han P, von Bergen M et al (2015) Cyanate as an energy source for nitrifiers. Nature 524: $105-108$

Palomo A, Fowler SJ, Gulay A, Rasmussen S, Sicheritz-Ponten T, Smets BF (2016) Metagenomic analysis of rapid gravity sand filter microbial communities suggests novel physiology of Nitrospira spp. ISME J 10:2569-2580

Palomo A, Pedersen AG, Fowler SJ, Dechesne A, Sicheritz-Ponten T, Smets BF (2017) Comparative genomics sheds light on niche differentiation and the evolutionary history of comammox Nitrospira. bioRxiv. https://doi.org/10.1101/138586

Park BJ, Park SJ, Yoon DN, Schouten S, Damste JSS, Rhee SK (2010) Cultivation of autotrophic ammonia-oxidizing archaea from marine sediments in coculture with sulfur-oxidizing bacteria. Appl Environ Microbiol 76:7575-7587

Pester M, Maixner F, Berry D, Rattei T, Koch H, Lucker S et al (2014) $\mathrm{NxrB}$ encoding the beta subunit of nitrite oxidoreductase as functional and phylogenetic marker for nitrite-oxidizing Nitrospira. Environ Microbiol 16:3055-3071

Pinto AJ, Marcus DN, Ijaz UZ, Bautista-de lose Santos QM, Dick GJ, Raskin L (2015) Metagenomic evidence for the presence of comammox Nitrospira-like bacteria in a drinking water system. mSphere 1:e00054-e0015

Pjevac P, Schauberger C, Poghosyan L, Herbold CW, van Kessel MAHJ, Daebeler A et al (2017) AmoA-targeted polymerase chain reaction primers for the specific detection and quantification of comammox Nitrospira in the environment. Front Microbiol 8:1508
Prosser JI (2011) Soil nitrifiers and nitrification. In: Ward BB, Arp DJ, Klotz MG (eds) Nitrification. ASM Press, Washington, DC, pp 347 383

Prosser JI, Nicol GW (2012) Archaeal and bacterial ammonia-oxidizers in soil: the quest for niche specialisation and differentiation. Trends Microbiol 20:523-531

Santoro AE (2016) The do-it-all nitrifier. Science 351:342-343

Shi XZ, Hu HW, Kelly K, Chen D, He JZ, Suter HC (2016a) Responses of ammonia oxidizers and denitrifiers to repeated applications of a nitrification inhibitor and a urease inhibitor in two pasture soils. J Soils Sediments 17:974-984

Shi XZ, Hu HW, Müller C, He JZ, Chen D, Suter HC (2016b) Effects of the nitrification inhibitor 3,4-dimethylpyrazole phosphate on nitrification and nitrifiers in two contrasting agricultural soils. Appl Environ Microbiol 82:5236-5248

Shi XZ, Hu HW, Zhu-Barker X, Hayden H, Wang JT, Suter H, Chen D, He JZ (2017) Nitrifier-induced denitrification is an important source of soil nitrous oxide and can be inhibited by a nitrification inhibitor 3,4-dimethylpyrazole phosphate. Environ Microbiol. https://doi.org/ $10.1111 / 1462-2920.13872$

Spang A, Poehlein A, Offre P, Zumbrägel S, Haider S, Rychlik N et al (2012) The genome of the ammonia-oxidizing Candidatus Nitrososphaera gargensis: insights into metabolic versatility and environmental adaptations. Environ Microbiol 14:3122-3145

Stieglmeier M, Mooshammer M, Kitzler B, Wanek W, ZechmeisterBoltenstem S, Richter A et al (2014) Aerobic nitrous oxide production through $\mathrm{N}$-nitrosating hybrid formation in ammonia-oxidizing archaea. ISME J 8:1135-1146

Stoecker K, Bendinger B, Schoning B, Nielsen PH, Nielsen JL, Baranyi $\mathrm{C}$ et al (2006) Cohn's Crenothrix is a filamentous methane oxidizer with an unusual methane monooxygenase. Proc Natl Acad Sci U S A 103:2363-2367

Tourna M, Stieglmeier M, Spang A, Könneke M, Schintlmeister A, Urich $\mathrm{T}$ et al (2011) Nitrososphaera viennensis, an ammonia oxidizing archaeon from soil. Proc Natl Acad Sci U S A 108:8420-8425

van Kessel MA, Speth DR, Albertsen M, Nielsen PH, den Camp HJO, Kartal B et al (2015) Complete nitrification by a single microorganism. Nature 528:555-559

Wang BZ, Zhao J, Guo Z, Ma J, Xu H, Jia Z (2015) Differential contributions of ammonia oxidizers and nitrite oxidizers to nitrification in four paddy soils. ISME J 9:1062-1075

Wang JT, Zheng YM, Hu HW, Li J, Zhang LM, Chen BD, Chen WP, He JZ (2016) Coupling of soil prokaryotic diversity and plant diversity across latitudinal forest ecosystems. Sci Rep 6:19561

Wang Y, Ma L, Mao Y, Jiang X, Xia Y, Yu K, Li B, Zhang T (2017) Comammox in drinking water systems. Water Res 116:332-341

Wrighton KC, Thomas BC, Sharon I, Miller CS, Castelle CJ, VerBerkmoes NC et al (2012) Fermentation, hydrogen, and sulfur metabolism in multiple uncultivated bacterial phyla. Science 337: 1661-1665

Zhang LM, Hu HW, Shen JP, He JZ (2012) Ammonia-oxidizing archaea have more important role than ammonia-oxidizing bacteria in ammonia oxidation of strongly acidic soils. ISME J 6:1032-1045 